\title{
Diretrizes
}

\section{Ecocardiografia em procedimentos intervencionistas}

A ecocardiografia é um instrumento indispensável não só para o diagnóstico, mas também no auxílio das intervenções terapêuticas percutâneas. É responsável pela identificação, seleção e monitoramento dos pacientes, assim como pelo seu acompanhamento tardio. Somado a isso, no laboratório de cateterismo cardíaco, seu uso também tem demonstrado uma redução do tempo de fluoroscopia e na quantidade de contraste utilizado, o que configura ganho secundário adicional da utilização dessa tecnologia ${ }^{1}$.

Os procedimentos intervencionistas que necessitam do apoio da ecocardiografia estão descritos na Tabela 36.

\section{1 - Punção transeptal}

A punção transeptal é utilizada quando o acesso ao átrio esquerdo é necessário. O exame transtorácico (ETT) ou a ecocardiografia transesofágica (ETE) auxilia esses procedimentos, permitindo a visibilização direta do cateter de punção transeptal e sua relação com a fossa oval ${ }^{4}$, diminuindo o risco de punção inadvertida da aorta intrapericárdica, da artéria pulmonar ou da parede atrial. A ecocardiografia

\section{TabelA 35 - Recomendações de monitorização ecocardiográfica (ETT ou ETE) na sala de hemodinâmica}

\begin{tabular}{|c|c|}
\hline Recomendações & Classe \\
\hline Punção transeptal & I \\
\hline Atriosseptostomia com cateter balão ou lâmina & 1 \\
\hline $\begin{array}{l}\text { Oclusão percutânea dos defeitos septais (comunicação interatrial, } \\
\text { forame oval patente e comunicações interventriculares) }\end{array}$ & I \\
\hline Procedimentos de ablação de arritmias por radiofrequência & I \\
\hline Pericardiocentese & I \\
\hline Biópsia miocárdica & I \\
\hline Valvoplastia percutânea com balão na estenose mitral (VPEM) & I \\
\hline $\begin{array}{l}\text { Alcoolização percutânea septal em miocardiopatia hipertrófica } \\
\text { obstrutiva }\end{array}$ & 1 \\
\hline Reparo percutâneo da valva mitral & I \\
\hline Implante percutâneo de endoprótese valvar & I \\
\hline $\begin{array}{l}\text { Outras situações: procedimentos para complementação da cirurgia } \\
\text { de Fontan (fechamento percutâneo de fenestrações com próteses); } \\
\text { procedimentos híbridos para implante de próteses intracardiacas } \\
\text { por punção direta do coração e oclusão percutânea do apêndice } \\
\text { atrial esquerdo em alguns casos de fibrilação atrial }\end{array}$ & I \\
\hline $\begin{array}{l}\text { Implante de endopróteses aórticas para tratamento de aneurismas, } \\
\text { dissecções, hematomas ou úlceras parietais da aorta torácica }{ }^{2}\end{array}$ & Ilb \\
\hline
\end{tabular}

Dependendo do procedimento ao qual o paciente será submetido, estará indicada a utilização do método transtorácico (ETT), transesofágico (ETE) ou intracardiaco (EIC) $)^{3}$. (Tabela 36). intracardíaca (EIC) oferece vantagens em relação à ETE, por não ser necessária a sedação do paciente e apresentar melhor resolução.

\section{2 - Atriosseptostomia com cateter balão ou lâmina}

A atriosseptostomia de Rashkind, realizada na sala de cateterismo como tratamento paliativo em crianças portadoras de transposição das grandes artérias, continua sendo amplamente empregada nos dias atuais. Esse procedimento, na maioria das vezes, é realizado na sala de hemodinâmica sob fluoroscopia, mas também pode ser executado à beira do leito na unidade de terapia intensiva neonatal, sob orientação exclusiva do ETT, evitando assim o transporte do neonato, cuja abordagem tem se mostrado segura e efetiva em vários estudos ${ }^{5}$.

\section{3 - Oclusão percutânea dos defeitos septais}

No fechamento das comunicações interatriais (CIA) por dispositivos introduzidos por via percutânea, é indispensável a ETE não só para a seleção de candidatos, mas, sobretudo, na sala de hemodinâmica, para posicionamento e liberação da prótese, além da avaliação imediata de fluxos residuais e o seguimento tardio ${ }^{6,7}$. As informações mais específicas

Tabela 36 - Recomendações das modalidades ecocardiográficas indicadas para o uso durante procedimentos intervencionistas

\begin{tabular}{|c|c|c|c|}
\hline Procedimento intervencionista & ETT & ETE & EIC \\
\hline Punção transeptal & + & ++ & ++ \\
\hline Valvoplastia percutânea com balão na EM (VPEM) & ++ & +++ & ++ \\
\hline $\begin{array}{l}\text { Oclusão percutânea de defeitos septais (CIA, FOP } \\
\text { e CIV) }\end{array}$ & + & +++ & ++ \\
\hline Alcoolização percutânea septal em CMH obstrutiva & ++ & ++ & - \\
\hline Reparo percutâneo da valva mitral & + & +++ & + \\
\hline $\begin{array}{l}\text { Assistência de implante percutâneo de dispositivo } \\
\text { no VE }\end{array}$ & - & ++ & ++ \\
\hline Implante percutâneo de endoprótese valvar aórtica & - & + & + \\
\hline Atriosseptostomia com balão ou lâmina & ++ & ++ & ++ \\
\hline Implante oclusor do apêndice atrial esquerdo & - & ++ & ++ \\
\hline Biópsia intravascular e miocárdica & ++ & ++ & ++ \\
\hline $\begin{array}{l}\text { Uso em cardiopatias congênitas (complementação } \\
\text { do procedimento de Fontan, correção de } \\
\text { coarctação da aorta }\end{array}$ & + & + & + \\
\hline Implante de endoprótese na aorta torácica & - & - & + \\
\hline
\end{tabular}

Legenda: (-) não documentado uso e benefício na literatura; (+) descrito uso e benefício existente, mas estudos futuros precisam ser delineados; (++) apresenta vantagens quando utilizado; $(+++)$ documentação clara quanto ao uso e benefício do método. CMHO - cardiomiopatia hipertrófica obstrutiva; CIA - comunicação interatrial; CIV - comunicação interventricular; EM - estenose mitral; FOP - forame oval patente. 
referentes às características dos defeitos, como número de orifícios, suas dimensões, localização no septo e características das bordas que os circundam, são mais bem avaliadas pela ecocardiografia transesofágica, que pode ser realizada ambulatorialmente ou imediatamente antes do implante, já na sala de hemodinâmica ${ }^{7}$. A realização do estudo transesofágico previamente ao dia do cateterismo traz vantagens quanto à programação do procedimento, seleção do dispositivo que será utilizado e aconselhamento familiar. O procedimento de oclusão da comunicação interatrial é continuamente monitorado pela ETE, podendo também ser realizada a partir da EIC e da ETE tridimensional (3D) em tempo real, este último permitindo uma análise extremamente detalhada da anatomia do septo interatrial ${ }^{8,9}$. Nos casos em que não seja possível a utilização da ETE para guiar a oclusão percutânea dos defeitos septais, está indicado o uso de $\mathrm{EIC}^{10}$.

Na oclusão percutânea da CIA, a ecocardiografia auxilia na medida do orifício e na escolha do número do dispositivo a ser empregado. Também monitora o posicionamento de guias, cateteres e bainhas no interior do coração e determina a disposição dos discos esquerdo e direito da prótese, antes e após a sua liberação ${ }^{11}$. Também se constitui em função primordial da ecocardiografia a análise cuidadosa da função das valvas aórtica e tricúspide e a detecção e graduação de possível fluxo residual.

12.4 - Estudos eletrofisiológicos para ablação por radiofrequência de focos arritmogênicos

Nos estudos eletrofisiológicos, a ecocardiografia intracardíaca é a modalidade ideal para monitorar e auxiliar tal procedimento ${ }^{8}$. Caso a EIC não esteja disponível, poderá ser substituída pela ETE. A utilização da ecocardiografia durante esse procedimento permite a realização da punção transeptal de forma segura, visibilização das veias pulmonares, posicionamento adequado do cateter no óstio da veia pulmonar - e não no seu interior -, monitora o aquecimento excessivo do tecido por meio da identificação de microbolhas e permite também a pesquisa de trombos, assim como o mapeamento completo do átrio esquerdo.

\section{5 - Pericardiocentese}

A eficácia e segurança da pericardiocentese guiada pela ecocardiografia tem sido demonstrada em vários subgrupos, incluindo os pacientes pediátricos ${ }^{12,13}$.

\section{6 - Biópsia miocárdica}

As biópsias endomiocárdicas são tipicamente realizadas no ventrículo direito para diagnosticar os vários tipos de desordens do miocárdio, incluindo cardiomiopatia infiltrativa e rejeição em transplante cardíaco ${ }^{14}$.

A ecocardiografia, particularmente o ETT, é uma modalidade de imagem muito útil nos procedimentos de biópsia intracardíaca e intravascular. Apesar da ETE e da EIC oferecerem imagens mais precisas que a ETT, os riscos adicionais e o custo mais alto destas modalidades fazem com que só sejam recomendadas em pacientes altamente selecionados.

\section{7 - Outras indicações da monitorização ecocardiográfica}

Na valvoplastia mitral percutânea, o ETT ou a ETE está indicada, pois auxilia na punção transeptal, pesquisa de trombo, avaliação dos gradientes e área valvar pré e pósprocedimento, assim como o reconhecimento precoce de possíveis complicações, como derrame pericárdico, formação de trombo, insuficiência mitral grave etc.

Recentemente, com o surgimento do implante percutâneo de endoprótese valvar aórtica, seja por via percutânea ou transapical, a ETE tem se mostrado de grande utilidade ${ }^{15}$. Nos reparos da valva mitral e na correção das deiscências protéticas, a ETE 3D também tem sido de grande valia.

$\mathrm{Na}$ ablação septal com álcool (ASA) dos casos de cardiomiopatia hipertrófica, a ecocardiografia (ETT ou ETE) com contraste (microbolhas, podendo também ser utilizado o próprio contraste radiológico agitado) está indicada, tendo durante o procedimento o objetivo primordial de detectar o ramo septal da descendente anterior esquerda que irriga a porção do septo interventricular em que ocorre o contato com a movimentação anterior da valva mitral.

Existem outras situações mais específicas em que a ecocardiografia está recomendada para monitorar procedimentos durante intervenções na sala de hemodinâmica, como na complementação da cirurgia de Fontan, em que está indicado o fechamento percutâneo de fenestrações com próteses; nos procedimentos híbridos para implante de próteses intracardíacas por punção direta do coração; e na oclusão percutânea do apêndice atrial esquerdo, em alguns casos de fibrilação atrial.

\section{8 - Usos emergentes}

Recentemente, tem sido descrito na literatura a utilização da ecocardiografia percutânea intrapericárdica (EPIP) em pacientes submetidos à ablação epicárdica de focos arritmogênicos, mostrando-se um método seguro e efetivo durante procedimentos eletrofisiológicos que envolvam acesso pericárdico. Tal acesso permite, em tempo real, imagens de alta resolução não habitualmente ou não facilmente obtidas com a EIC, devido à interferência dos cateteres utilizados na ablação ${ }^{8}$.

A nova aplicação da imagem tridimensional (3D) na ecocardiografia oferece uma contribuição significativa nos procedimentos intervencionistas. Quando a imagem 3D é utilizada na biópsia miocárdica, o procedimento fica mais seguro e eficaz ${ }^{16}$.

Em alguns centros, a sonda esofágica tridimensional em tempo real (ETE 3D) já está disponível. Trata-se de uma ferramenta que vem acrescentar um ganho importante à ecocardiografia, podendo vir a ser o método preferencial, quando disponível, em diversas situações, como, por exemplo, na oclusão percutânea de defeitos septais, principalmente nas comunicações interatriais e no reparo percutâneo da valva mitral. 


\section{Diretrizes}

\section{Referências}

1. Van der Velde ME, Perry SB. Transesophageal echocardiography during interventional catheterization in congenital heart disease. Echocardiography. 1997; 14: 513-28.

2. Fischer $\mathrm{CH}$, Campos FO, Fonseca JHAP, Alves CMR, Sousa JAM, de Lira Filho EB, et al. Utilização da ecocardiografia transesofágica durante implante de endoprótese aórtica (stent). Experiência inicial. Arq Bras Cardiol. 2001; 77: 1-4.

3. Silvestry FE, Kerber RE, Brook MM, Carroll JD, Eberman KM, Goldstein $\mathrm{SA}$, et al. ASE recommendations for clinical practice: echocardiography: guided interventions. J Am Soc Echocardiogr. 2009; 22 (3): 213-31.

4. Hurrell DG, Nishimura RA, Symanski JD,Holmes DR. Echocardiography in the invasive laboratory: utility of two-dimensional echocardiography in performing transseptal catheterization. Mayo Clin Proc. 1998; 73: 126-31.

5. Kakadekar AP, Hayes A, Rosenthal E, Huggon IC, Baker EJ, Qureshi $\mathrm{SA}$, et al. Balloon atrial septostomy in the intensive care unit under echocardiographic control: nine years experience. Cardiol Young. 1992; 2: $175-8$.

6. Fischer G, Stieh J, Uebing A, Hoffmann U, Morf G, Kramer HH. Experience with transcatheter closure of secundum atrial septal defects using the Amplatzer septal occluder: a single centre study in 236 consecutive patients. Heart. 2003; 89: 199-204.

7. Pedra SR, Pedra CA, Assef JE, Cassar RS, Esteves CA, Braga SN, et al. Percutaneous closure of atrial septal defects. The role of transesophageal echocardiography. Arq Bras Cardiol. 1999; 72 (1): 59-69.

8. Hijazi ZM, Shivkumar K, Sahn DJ. Intracardiac echocardiography during interventional and electrophysiological cardiac catheterization. Circulation. 2009; 119: 587-96.

\section{Cardiologia fetal, cardiologia pediátrica e cardiopatias congênitas do adulto}

\section{1 - Recomendações para ecocardiografia fetal}

A ecocardiografia fetal é ferramenta fundamental para o diagnóstico cardiológico intrauterino ${ }^{1-8}$. Muitas cardiopatias graves, com necessidade de atendimento clínico-cirúrgico de emergência logo após o nascimento, passaram a ter seu diagnóstico conhecido ainda na vida intrauterina, propiciando o planejamento antecipado das ações a serem adotadas pela equipe médica no pós-parto imediato ou mesmo durante a vida fetal ${ }^{1,5,6}$. O exame pode ser realizado no primeiro trimestre, tanto por via transvaginal como transabdominal ${ }^{7}$. Entretanto, é a partir da $18^{\underline{a}}$ semana de gestação até o termo que a maioria das alterações estruturais ou funcionais do coração fetal podem ser identificadas. O ritmo cardíaco fetal deve ser
9. Marx GR, Sherwood MC, Fleishman C, Van Praagh R. Three-dimensional echocardiography or the atrial septum. Echocardiography. 2001; 18 (5): 433-43.

10. Zanchetta M, Rigatelli G, Pedon L, Zennaro M, Carrozza A, Onorato E, et al. Transcatheter atrial septal defect closure assisted by intracardiac echocardiography: 3-year follow-up. J Interv Cardiol. 2004: 17 (2): 95-8.

11. Pedra CA, Pedra SR, Esteves CA, Pontes SC Jr, Braga SL, Arrieta SR, et al. Percutaneous closure of perimembranous ventricular septal defects with the Amplatzer devide: technical and morphological considerations. Catheter Cardiovasc Interv. 2004; 61 (3): 403-10.

12. Tsang T, El-Najdawi E, Freeman W, Hagler D, Seward J, O'Leary P. Percutaneous echocardiographically guided pericardiocentesis in pediatric patients: evaluation of safety and efficacy. J Am Soc Echocardiogr. 1998; 11: 1072-7.

13. Cauduro S, Moder K, Tsang T, Seward J. Clinical and echocardiographic characteristics of hemodynamically significant pericardial effusions in patients with systemic lupus erythematosus. Am J Cardiol. 2003; 92: 1370-2.

14. Ragni T, Martivelli L, Goggi C, Speziali G, Rinaldi M, Roda G, et al. Echocontrolled endomyocardial biopsy. J Heart Transplant. 1990; 9: 538-42.

15. Bleiziffer S, Ruge H, Mazzitelli D, Schreiber C, Hutter A, Laborde JC, et al. Results of percutaneous and transapical transcatheter aortic valve implantation performed by a surgical team. Eur J Cardiothoracic Surg. 2009; 35 (4): 615-20.

16. McCreery CJ, McCulloch M, Ahmad M, de Filippi CR. Real-time 3-dimensional echocardiography imaging for right ventricular endomyocardial biopsy: a comparison with fluoroscopy. J Am Soc Echocardiogr. 2001; 14: 927-33.

avaliado em todas as gestantes, na busca da detecção de arritmias graves e potencialmente fatais, que irão necessitar de tratamento e acompanhamento pré-natal, assim como de sua diferenciação com arritmias de menor significado clínico. A avaliação da função cardíaca fetal, tanto sistólica como diastólica, é essencial em situações de doenças miocárdicas primárias ou secundárias, tal como ocorre no diabetes materno ${ }^{9}$.

Quando detectada uma cardiopatia estrutural, especialmente se complexa, um importante incremento nas informações morfofuncionais pode ser obtido com o ecocardiograma tridimensional (3D) e o tetradimensional (4D), utilizando a tecnologia STIC (spatio-temporal image correlation, ou correlação têmporo-espacial da imagem $)^{10,11}$.

As indicações clínicas e as recomendações para ecocardiografia fetal estão sumarizadas na Tabela 37. 